\section{Evaluación de un programa ministerial para manejo del síndrome metabólico en adultos con sobrepeso y obesidad}

\author{
SANDRA HENRÍQUEZ, GLADYS BARRERA ${ }^{\mathrm{a}}$, \\ SANDRA HIRSCH, MARÍA P. DE LA MAZA, \\ NATALIA JARA, LAURA LEIVA ${ }^{\mathrm{b}}$, DANIEL BUNOUT
}

\section{Evaluation of a program for management of metabolic syndrome in adults with overweight and obesity}

\begin{abstract}
Background: The Chilean Ministry of Health developed a healthy lifestyles intervention directed to adults with overweight and cardiovascular risk factors, called "Program on Healthy Eating and Physical Activity" (PASAF). Aim: To evaluate the impact of PASAF on nutritional status and metabolic parameters. Patients and Methods: We analyzed databases from three primary care centers belonging to a municipality of Metropolitan Santiago. We selected adults enrolled in the PASAF during three years (2007-2009). The program lasted four months and included an assessment of anthropometric and metabolic parameters at baseline and at the end, eight workshops with a nutritionist, seven with a psychologist and 32 sessions of physical activity. Results: We evaluated 526 subjects aged $\geq 18$ years (93\% females), of whom $85.6 \%$ attended the last appointment for assessment. Analyzing available data, attendance to workshops was $<50 \%$ of the scheduled sessions. Weight, body mass index and waist circumference decreased significantly (median: $-1.4 \mathrm{~kg},-0.6 \mathrm{~kg} / \mathrm{m}^{2}$ and $-3 \mathrm{~cm}$, respectively). The median weight loss was $1.8 \%$ of initial weight and $17.1 \%$ of participants experienced a decrease $\geq 5 \%$ of their initial weight. There were significant improvements in lipid levels and blood pressure among participants with lower initial excess weight. A reduction in fasting blood glucose was observed only among subjects who lost $\geq 5 \%$ of their initial weight. Conclusions: The PASAF modestly reduced nutritional parameters. Correction of metabolic parameters was especially effective in less obese subjects. The attendance to workshops was low.
\end{abstract}

(Rev Med Chile 2014; 142: 817-825)

Key words: Life style; Metabolic Syndrome X; Obesity.

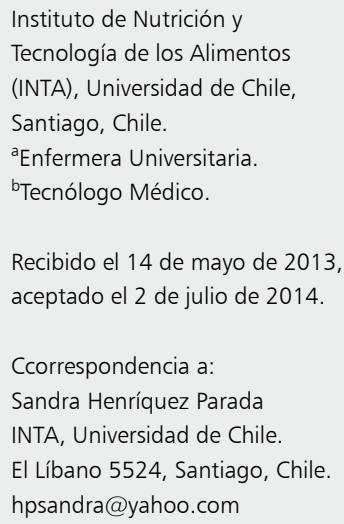

$\tau$ a prevalencia de obesidad ha ido en aumento a nivel mundial, contribuyendo al desarrollo de enfermedades crónicas no transmisibles $(\mathrm{ECNT})^{1}$. En Chile, la última encuesta nacional de salud (ENS) realizada en 2009-2010 encontró altas prevalencias de exceso de peso $(64,5 \%)$, sedentarismo (88,6\%), síndrome metabólico (35\%) y $\mathrm{ECNT}^{2}$.

A nivel mundial se han implementado diversos programas en atención primaria de salud (APS), dirigidos a cambios de estilo de vida saludable y reducción de la ingesta calórica, pero pocos estudios han revisado los resultados obtenidos. Wadden y col. analizaron una intervención realizada en 6 centros de APS durante dos años, basada en dieta y actividad física, encontrando que el grupo que recibió visitas trimestrales para entrega de recomendaciones sobre control de peso $(n=130)$, disminuyó 
en promedio $1,7 \mathrm{~kg}^{3}$. Jebb y col. también encontraron resultados positivos al analizar la efectividad de un programa basado en educación sobre estilos de vida saludables, aplicado a 395 sujetos pertenecientes a centros de APS de Australia, Alemania y el Reino Unido durante un año, encontrando una disminución de peso promedio de $2,3 \mathrm{~kg}^{4}$. Sin embargo, otros estudios similares no han encontrado resultados tan favorables, como los observados por Admiraal y col. en una intervención durante 1 año en sujetos sudasiáticos con riesgo de desarrollar diabetes mellitus 2 (DM2), donde el grupo intervenido con cambios de estilo de vida saludables $(n=177)$ no presentó mejorías significativas en el peso ni en el perfil metabólico respecto al grupo control $(\mathrm{n}=158)^{5}$; o los resultados del Programa Nacional de Prevención de Diabetes en 400 centros de APS de Finlandia durante los años 2003-2008, donde la disminución de peso y de circunferencia de cintura (CC) fueron sólo de $1 \mathrm{~kg}$ y de $1,3 \mathrm{~cm}$, respectivamente ${ }^{6}$.

En Chile, el Ministerio de Salud (MINSAL), desarrolló una intervención en cambios de estilo de vida saludables llamado "Programa de Alimentación Saludable y Actividad Física-Adultos" (PASAF), dirigido a adultos con índice de masa corporal (IMC) entre 25 y $45 \mathrm{~kg} / \mathrm{m}^{2}$, y uno o más de los siguientes factores de riesgo: dos glicemias de ayuno alteradas (100-125 mg/dL), prehipertensión (presión sistólica entre 130-139 mmHg o diastólica entre 85-89 mmHg), o antecedentes de DM2 en un familiar de primer grado.

El objetivo general del programa fue disminuir los factores de riesgo cardiovascular asociados al síndrome metabólico para contribuir a la prevención de ECNT, y los objetivos específicos contemplaron: mejorar el estado nutricional (IMC, CC); mejorar las condiciones asociadas (hipertensión arterial, dislipidemia, intolerancia a la glucosa) y mejorar la condición física (capacidad funcional muscular y aeróbica).

El programa duró cuatro meses, e incluyó:

- 6 consultas individuales: 1 por médico, 1 por psicólogo y 4 por nutricionista.

- 15 encuentros grupales: 7 por psicólogo y 8 por nutricionista.

- 32 sesiones de actividad física.

- Al inicio y al término: antropometría, glicemia en ayunas, triglicéridos y colesterol $\mathrm{HDL}^{7}$.

Este programa comenzó como piloto el año 2004, incluyendo 276 sujetos, de los cuales 58\% completó el programa y 55\% redujo $\geq 5 \%$ de su peso inicial, con una mediana de disminución de peso de 3,9\%; además consiguió reducciones significativas en los parámetros antropométricos y metabólicos (glicemia, insulinemia, HOMA y colesterol HDL $)^{8}$. El programa fue ampliando su cobertura, extendiéndose a todos los servicios de salud del país el año $2007^{9-10}$. A lo largo del tiempo se realizaron modificaciones con el fin de reducir costos, disminuyendo el número de exámenes y consultas médicas ${ }^{11}$. En 2008 se evaluó el programa a nivel nacional, encontrándose resultados positivos tanto en la mejoría clínica como en la adherencia $^{9-11}$. En año 2011, el MINSAL resolvió crear un programa piloto preventivo basado en el PASAF, para dar respuesta a la alta prevalencia de obesidad y ECNT ${ }^{12}$. El objetivo de este estudio es evaluar el impacto del PASAF sobre los parámetros nutricionales y metabólicos en 3 consultorios de la comuna de Macul, para así contribuir a la evaluación del programa.

\section{Material y Método}

Se realizó un estudio longitudinal descriptivo, utilizando los registros del PASAF adultos de los centros de APS de la comuna de Macul (Santa Julia, Padre Hurtado y Félix de Amesti), recopilados en planillas Excel entre los años 2007 y 2009. Para estimar la adherencia se consideró a los individuos que contaron con registro antropométrico al inicio y al cuarto mes. Se analizaron las variables antropométricas (peso, IMC, CC), presión arterial (PA) e indicadores bioquímicos de riesgo cardiovascular (glicemia, colesterol total, colesterol HDL y triglicéridos) al inicio y final del período de intervención.

Para analizar los cambios en las variables antropométricas, se utilizaron los siguientes puntos de corte:

\section{Estado nutricional}

Clasificación de la OMS para IMC: bajo peso $<18,5 \mathrm{~kg} / \mathrm{m}^{2}$, normal $18,5-24,9 \mathrm{~kg} / \mathrm{m}^{2}$, sobrepeso $25-29,9 \mathrm{~kg} / \mathrm{m}^{2}$, obesidad clase I $30-34,9 \mathrm{~kg} / \mathrm{m}^{2}$, obesidad clase II $35-39,9 \mathrm{~kg} / \mathrm{m}^{2}$, obesidad clase III $\geq 40 \mathrm{~kg} / \mathrm{m}^{213}$.

\section{Obesidad abdominal}

Se consideraron alteradas la CC $>102 \mathrm{~cm}$ en hombres y $>88 \mathrm{~cm}$ en mujeres ${ }^{7}$. 


\section{Estadística}

Se evaluó la normalidad de las variables a través del test Shapiro-Wilk, y posteriormente se utilizaron tests no paramétricos. Se realizó un análisis descriptivo de los datos con estadística básica. Se empleó test de simetría para analizar las diferencias de proporciones entre el inicio y el final para IMC y CC. Se estudiaron los cambios en los parámetros antropométricos, PA e indicadores bioquímicos utilizando Signrank test de Wilcoxon, para el total de la muestra y para subgrupos según: estado nutricional inicial y porcentaje de disminución de peso. Los análisis se realizaron con el programa computacional STATA 10.0, utilizando nivel de significación estadística de $95 \%$.

\section{Resultados}

\section{Características de la muestra al ingreso}

Entre los años 2007 y 2009 ingresaron al programa 526 sujetos ( $93,3 \%$ mujeres), cuyas características generales se describen en la Tabla 1 . De la totalidad de la muestra, $34,2 \%$ presentó sobrepeso y $65,8 \%$ obesidad (39\% clase I, $18,8 \%$ clase II y $8 \%$ clase III). El 57,4\% ingresó por tener "antecedente

Tabla 1. Características generales de la muestra al ingreso (n 526)

\begin{tabular}{|lccc|}
\hline Variables & n & Mediana & (p25-p75) \\
Mujer/Hombre & 526 & $491 / 35$ & \\
Edad (años) & 526 & 40,5 & $(30-48)$ \\
Peso inicial (kg) & 526 & 78,8 & $(70,4-88,6)$ \\
IMC (kg/m²) & 526 & 31,4 & $(29-35,6)$ \\
CC Hombre (cm) & 34 & 108,5 & $(101-116)$ \\
CC Mujer (cm) & 484 & 100 & $(93-109)$ \\
Glicemia de ayuno (mg/dL) & 360 & 91 & $(84-98)$ \\
Colesterol total (mg/dL) & 357 & 196 & $(167-226)$ \\
HDL Hombre (mg/dL) & 19 & 40 & $(34-51)$ \\
HDL Mujer (mg/dL) & 296 & 50 & $(40-62)$ \\
LDL (mg/dL) & 301 & 114 & $(91-138)$ \\
TG (mg/dL) & 347 & 129 & $(91-195)$ \\
PA sistólica (mmHg) & 397 & 120 & $(110-128)$ \\
PA diastólica (mmHg) & 398 & 80 & $(70-80)$ \\
\hline
\end{tabular}

IMC: índice de masa corporal; CC: Circunferencia de cintura; TG: triglicéridos; PA presión arterial; p25: Percentil 25; p75: Percentil 75. *Nota: Los (n) de las distintas variables cambian según la disponibilidad de datos. familiar de DM2", 12,2\% por prehipertensión, $5,7 \%$ por 2 glicemias de ayuno alteradas. No se registró la causa de ingreso en $24,7 \%$ de los casos.

\section{Registro de datos}

De los sujetos que completaron el programa $(\mathrm{n}=450)$, se observó déficit en el registro de la PA e indicadores bioquímicos: no se contó con el registro de la PA en $20 \%$ de los casos al inicio y $52 \%$ al final; no se registraron los exámenes de laboratorio en $31 \%$ de los sujetos al inicio y $68 \%$ al final.

Respecto a la asistencia a talleres, no se contó con registro de actividad física, nutrición y psicología en $24 \%, 45 \%$ y $43 \%$ de los sujetos que completaron el programa, respectivamente.

\section{Adherencia}

Acudieron al último control 450 sujetos, lo que corresponde a $85,6 \%$ de la muestra. De ellos, la asistencia promedio a talleres de actividad física fue de 9 sesiones (28\%), a nutricionista fue de 3,3 sesiones (41\%) y a psicólogo fue de 2,7 sesiones (39\%), considerando a los participantes que contaban con dicho registro) (Figura 1). Los pacientes que no completaron el seguimiento (n = 76) tuvieron similar edad y exceso de peso que los que acudieron al último control, pero hubo una mayor proporción de hombres (13\% versus $6 \%$, respectivamente).

\section{Cambios en la condición nutricional}

De los individuos que completaron el programa $(\mathrm{n}=450), 71,5 \%$ disminuyó de peso, pero sólo

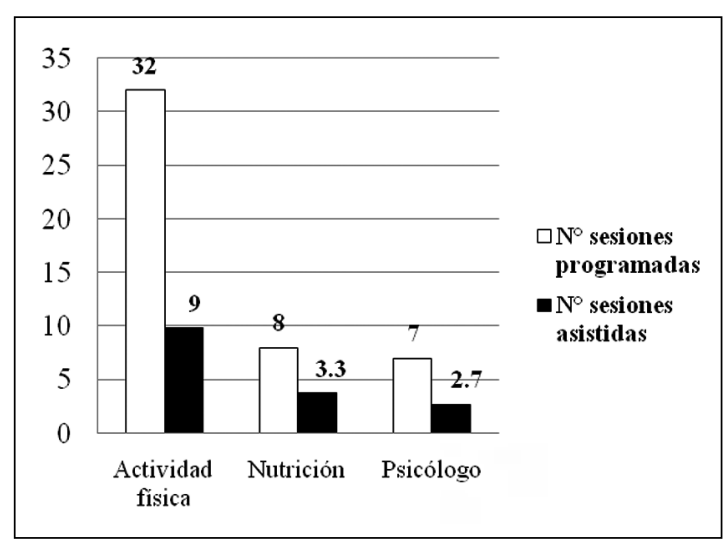

Figura 1. Número de sesiones programadas versus promedio de sesiones asistidas, en los sujetos que completaron el programa $(n=450)$. 


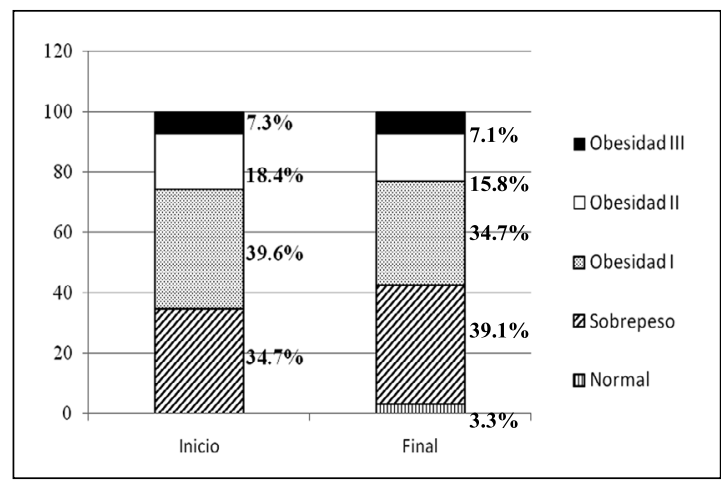

Figura 2. Estado nutricional de los participantes que completaron el programa $(n=450)$

$17,1 \%$ redujo $\geq 5 \%$ de su peso inicial. La mediana de reducción de peso fue de $1,8 \%$ respecto al peso de ingreso.

El porcentaje de sujetos con obesidad disminuyó en 7,7 puntos porcentuales al término del programa $(\mathrm{p}<0,05)$. La Figura 2 muestra el porcentaje de disminución de sujetos con obesidad clase I y II $(-4,9$ y $-2,6$ puntos porcentuales respectivamente $(\mathrm{p}<0,05))$. Sin embargo, el porcentaje de sujetos con obesidad clase III se mantuvo cercano a 7\% (no significativo).

El porcentaje de sujetos con obesidad abdo- minal disminuyó de $87,4 \%$ a $77,7 \%$ (-9,7 puntos porcentuales $(\mathrm{p}<0,05))$.

Para los sujetos que completaron el programa, el peso, el IMC y la CC presentaron reducciones significativas (medianas: $-1,4 \mathrm{~kg},-0,6 \mathrm{~kg} / \mathrm{m}^{2}$ y $-3 \mathrm{~cm}$ respectivamente) (Tabla 2). Al analizar a los sujetos según su estado nutricional inicial, las reducciones siguieron siendo significativas para todos los grupos (Tabla 3).

\section{Cambios en los indicadores bioquímicos y PA}

Para analizar los cambios en los indicadores bioquímicos y PA, se consideró sólo a los sujetos que completaron el programa y que tuvieron registro de exámenes de laboratorio tanto inicial como final. Se observaron mejorías significativas para el colesterol total, LDL, HDL (Tabla 2), y al realizar el análisis según el estado nutricional inicial (Tabla 3), se observaron mejorías significativas en:

- Colesterol total y LDL en los sujetos con sobrepeso al ingreso.

- Colesterol LDL, HDL y PA sistólica (con valor límite para la PA diastólica) en los sujetos con obesidad clase I al ingreso.

- En los grupos con obesidad clase II o III al ingreso, no se observaron cambios significativos para ninguno de los indicadores bioquímicos ni para la $\mathrm{PA}$.

Tabla 2. Cambios en los parámetros antropométricos, metabólicos y PA en los sujetos que completaron el programa $(n=450)$

\begin{tabular}{|c|c|c|c|c|c|c|c|}
\hline Variables & $\mathbf{n}$ & Mediana & $\begin{array}{l}\text { cio } \\
\text { (p25-p75) }\end{array}$ & Mediana & $\begin{array}{l}\text { Ial } \\
\text { (p25-p75) }\end{array}$ & $\mathbf{p}$ & $\begin{array}{c}\text { Mediana de } \\
\text { cambio }\end{array}$ \\
\hline Peso $(k g)$ & 450 & 78 & $(70-88)$ & 76 & $(68-86,7)$ & $<0,05$ & $-1,4$ \\
\hline $\mathrm{CC}(\mathrm{cm})$ & 443 & 100 & (94-109) & 97 & $(90-105)$ & $<0,05$ & -3 \\
\hline IMC (kg/m²) & 450 & 31,3 & $(29-35,3)$ & 30,8 & $(28,5-34,7)$ & $<0,05$ & $-0,6$ \\
\hline Gli ay (mg/dL) & 133 & 90 & $(84-97,5)$ & 88,5 & $(84-96)$ & NS & 0 \\
\hline Col total (mg/dL) & 138 & 202 & $(171-238)$ & 192 & $(164-226)$ & $<0,05$ & -7 \\
\hline Col LDL (mg/dL) & 115 & 119 & $(96-142)$ & 105 & $(81-129)$ & $<0,05$ & -11 \\
\hline Col HDL (mg/dL) & 122 & 48,5 & $(40-59)$ & 52 & $(41-63)$ & $<0,05$ & 2,5 \\
\hline TG (mg/dL) & 135 & 135 & $(87-215)$ & 151 & $(96-199)$ & NS & 1 \\
\hline PAS (mmHg) & 215 & 120 & $(110-125)$ & 120 & $(110-124)$ & NS & 0 \\
\hline PAD $(\mathrm{mmHg})$ & 215 & 80 & $(70-80)$ & 80 & $(70-80)$ & NS & 0 \\
\hline
\end{tabular}

p25: percentil 25; p75: percentil 75; N.S: no significativo; CC: circunferencia de cintura; IMC: índice de masa corporal; Gli ay: glicemia ayuno; Col: colesterol; TG: triglicéridos; PAS: presión arterial sistólica; PAD: presión arterial diastólica. Test de Wilcoxon para cambios intragrupo. *Nota: Los (n) de las distintas variables cambian según la disponibilidad de datos. 


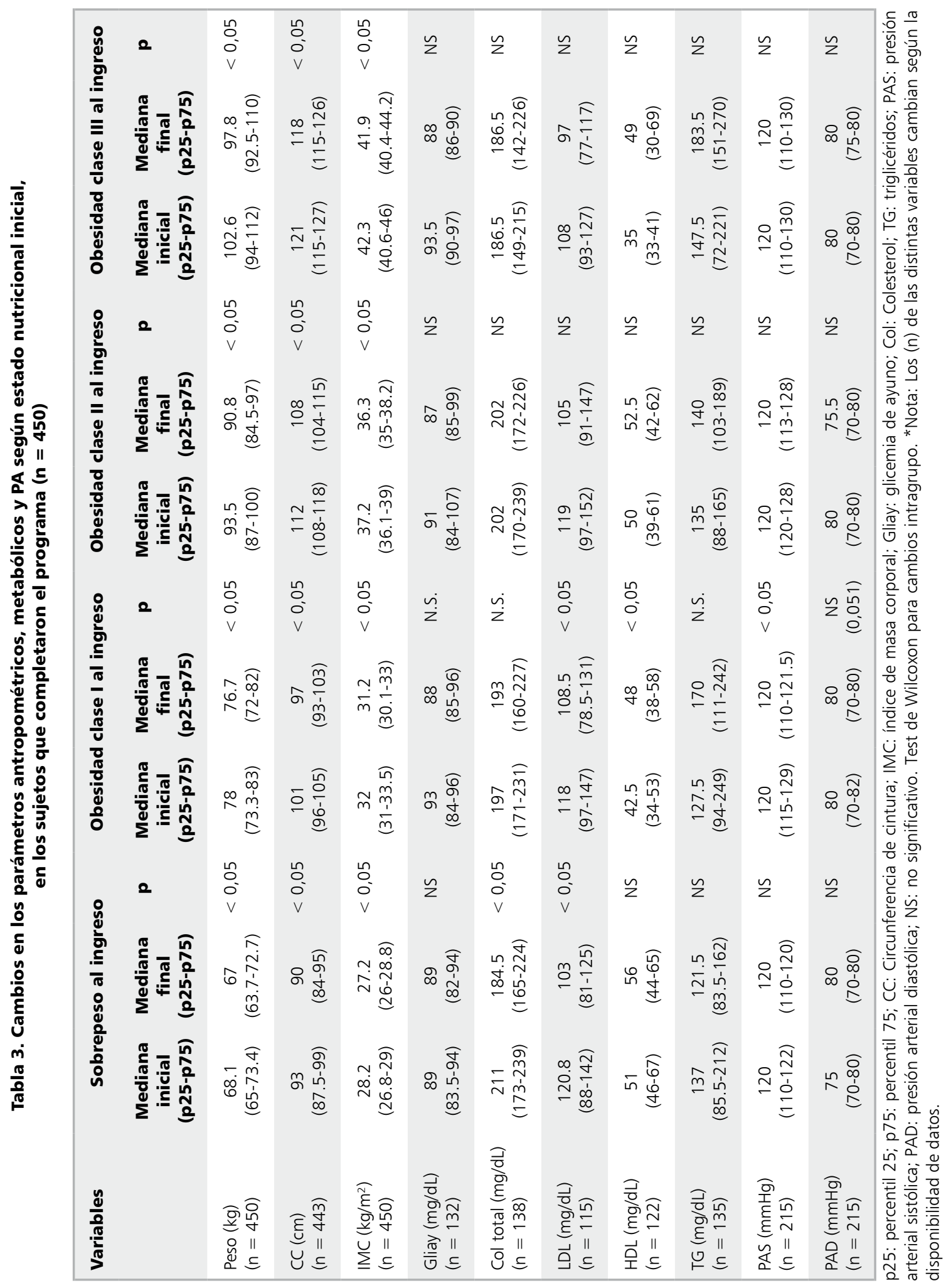


Tabla 4. Cambio en los indicadores bioquímicos y PA en función del porcentaje de cambio de peso, en los sujetos que completaronvel programa $(n=450)$

\begin{tabular}{|c|c|c|c|c|c|c|}
\hline \multirow[t]{2}{*}{ Variables } & \multicolumn{3}{|c|}{$\downarrow \geq \mathbf{5} \%$ peso inicial } & \multicolumn{3}{|c|}{ NO $\downarrow \geq \mathbf{5} \%$ peso inicial } \\
\hline & $\begin{array}{l}\text { Mediana inicial } \\
\text { (p25-p75) }\end{array}$ & $\begin{array}{l}\text { Mediana final } \\
(\text { p25-p75) }\end{array}$ & $\mathbf{p}$ & $\begin{array}{l}\text { Mediana inicial } \\
\text { (p25-p75) }\end{array}$ & $\begin{array}{l}\text { Mediana final } \\
\text { (p25-p75) }\end{array}$ & $\mathbf{p}$ \\
\hline $\begin{array}{l}\text { CC }(\mathrm{cm}) \\
(\mathrm{n}=443)\end{array}$ & $\begin{array}{c}101 \\
(93-109)\end{array}$ & $\begin{array}{c}92 \\
(86-100)\end{array}$ & $<0,05$ & $\begin{array}{c}100 \\
(94-110)\end{array}$ & $\begin{array}{c}98 \\
(92-106)\end{array}$ & $<0,05$ \\
\hline $\begin{array}{l}\text { Gli ay }(\mathrm{mg}(\mathrm{dL}) \\
(\mathrm{n}=132)\end{array}$ & $\begin{array}{c}91 \\
(85-99)\end{array}$ & $\begin{array}{c}87 \\
(83-94)\end{array}$ & $<0,05$ & $\begin{array}{c}90 \\
(84-97)\end{array}$ & $\begin{array}{c}89 \\
(84-96)\end{array}$ & NS \\
\hline $\begin{array}{l}\text { Col total }(\mathrm{mg} / \mathrm{dL}) \\
(\mathrm{n}=138)\end{array}$ & $\begin{array}{c}203 \\
(172-221)\end{array}$ & $\begin{array}{c}186 \\
(147-223)\end{array}$ & $<0,05$ & $\begin{array}{c}201 \\
(171-238)\end{array}$ & $\begin{array}{c}193 \\
(166-226)\end{array}$ & $<0,05$ \\
\hline $\begin{array}{l}\mathrm{LDL}(\mathrm{mg} / \mathrm{dL}) \\
(\mathrm{n}=115)\end{array}$ & $\begin{array}{c}116 \\
(103-149)\end{array}$ & $\begin{array}{c}104 \\
(83-137)\end{array}$ & $<0,05$ & $\begin{array}{c}120 \\
(93-142)\end{array}$ & $\begin{array}{c}106 \\
(76-128)\end{array}$ & $<0,05$ \\
\hline $\begin{array}{l}\mathrm{HDL}(\mathrm{mg} / \mathrm{dL}) \\
(\mathrm{n}=122)\end{array}$ & $\begin{array}{c}42 \\
(34-52)\end{array}$ & $\begin{array}{c}45 \\
(38-58)\end{array}$ & NS & $\begin{array}{c}50 \\
(40-62)\end{array}$ & $\begin{array}{c}54 \\
(44-64)\end{array}$ & $<0,05$ \\
\hline $\begin{array}{l}\text { TG }(m g / d L) \\
(n=135)\end{array}$ & $\begin{array}{c}126 \\
(91-210)\end{array}$ & $\begin{array}{c}113 \\
(83-171)\end{array}$ & NS & $\begin{array}{c}137 \\
(87-215)\end{array}$ & $\begin{array}{c}158 \\
(104-200)\end{array}$ & NS \\
\hline $\begin{array}{l}\text { PAS }(\mathrm{mmHg}) \\
(\mathrm{n}=215)\end{array}$ & $\begin{array}{c}120 \\
(115-128)\end{array}$ & $\begin{array}{c}120 \\
(110-120)\end{array}$ & NS & $\begin{array}{c}120 \\
(110-125)\end{array}$ & $\begin{array}{c}120 \\
(110-125)\end{array}$ & NS \\
\hline $\begin{array}{l}\text { PAD }(\mathrm{mm} / \mathrm{Hg}) \\
(\mathrm{n}=215)\end{array}$ & $\begin{array}{c}80 \\
(70-82)\end{array}$ & $\begin{array}{c}80 \\
(75-80)\end{array}$ & NS & $\begin{array}{c}80 \\
(70-80)\end{array}$ & $\begin{array}{c}80 \\
(70-80)\end{array}$ & NS \\
\hline
\end{tabular}

p25: percentil 25; p75: percentil 75; NS: No significativo; CC: Circunferencia de cintura; Gli ay: glicemia de ayuno; Col: colesterol; TG: triglicéridos; PAS: presión arterial sistólica; PAD: presión arterial diastólica. Test de Wilcoxon para cambios intragrupo. *Nota: Los ( $\mathrm{n}$ ) de las distintas variables cambian según la disponibilidad de los datos.

La Tabla 4 muestra los cambios en los parámetros metabólicos en función del porcentaje de disminución de peso, apreciándose mejorías significativas en:

- La CC, el colesterol total y LDL para todos los sujetos.

- La glicemia en el grupo que disminuyó $\geq 5 \%$ de su peso inicial.

\section{Discusión}

Este estudio encontró que los sujetos que acudieron al término del PASAF mejoraron su condición nutricional en forma modesta, en relación a los resultados obtenidos en otras evaluaciones sobre el programa ${ }^{8,11}$.

En los tratamientos para obesidad, el abandono es uno de los principales problemas, esencialmente debido a la expectativa de una mayor pérdida de peso inicial ${ }^{8,14}$. Evaluaciones previas del PASAF (2004, 2008) encontraron bajos porcentajes de sujetos que llegan al término del programa (cercanos a $60 \%)^{8,11}$, similar a los resultados encontrados en otras intervenciones internacionales, como la realizada en centros de APS de Australia, Alemania y Reino Unido, donde sólo 54\% completó los 12 meses de duración ${ }^{4}$. A pesar de que en este estudio un alto porcentaje de individuos acudió al término del programa $(86 \%)$, la asistencia registrada a los talleres y actividad física fue baja $(<50 \%$ de las sesiones programadas), resultado similar al observado en la evaluación del PASAF realizada en la Región de Los Ríos el año 2008, que describe que la asistencia promedio a talleres de nutrición fue de $54 \%$ y a psicólogo sólo $26 \%$, concluyendo además que la obtención de mejores resultados podrían lograrse a través de una mayor asistencia a talleres y ejercicio ${ }^{15}$. Los resultados modestos obtenidos en este estudio podrían ser explicados por la baja asistencia a los talleres, ya que en general la literatura demuestra que un mayor número de atenciones se asocia a mayor pérdida de peso ${ }^{3}$. El problema de la baja adherencia a este tipo de programas es 
común de observar, como podemos apreciar en un estudio realizado por Arrebola E y cols. sobre 60 sujetos con exceso de peso, que encontró $45 \%$ de adherencia (definida como asistencia $\geq 80 \%$ de las sesiones programadas $)^{16}$.

Es sabido que en obesidad la pérdida $\geq 5 \%$ del peso se asocia a mejorías en los parámetros metabólicos ${ }^{17,18}$, siendo considerada como la variable de resultado principal en los tratamiento para obesidad según la "US. Food and Drug Administration"19. En este estudio, menos de un quinto de los sujetos disminuyó dicho porcentaje de peso; resultado similar al encontrado en el análisis sobre el PASAF realizado en la Región de los Ríos (ya mencionado), donde $24,2 \%$ disminuyó $\geq 5 \%$ del peso; resultados similares al encontrado en una intervención sobre cambios de estilo de vida saludables realizada por la Universidad de Pensilvania en seis centros de APS durante dos años, donde $21,5 \%$ redujo $\geq 5 \%$ del peso $^{3}$. No obstante, otras intervenciones han encontrado mejores resultados, como en un estudio realizado en Finlandia durante 1 año en sujetos prediabéticos donde $43 \%$ logró perder $\geq 5 \%$ del peso ${ }^{20}$. Asimismo, en la evaluación nacional del PASAF publicada el año 2008, 40\% disminuyó $\geq 5 \%$ de su peso $^{11}$ y en el piloto del programa (año 2004) 55\% redujo este porcentaje, con medianas de cambio de peso e IMC tres veces superiores a las halladas en este estudio ${ }^{8}$. Es posible que la diferencia en los resultados se deba a que este estudio incluyó a participantes con obesidad mórbida, en quienes las intervenciones no quirúrgicas son menos exi$\operatorname{tosas}^{21,22}$. Otros factores que pueden explicar esta diferencia son: la baja adherencia a los talleres y el menor número de controles por médico (uno) en relación al programa piloto (tres), pudiendo esto influir en una mayor motivación en los participantes ${ }^{8}$.

La leve disminución de peso observada en este programa fue mayor a la que se encuentra en grupos no tratados. El estudio de Bennet y cols. muestra que obesos de nivel socioeconómico medio-bajo, seguidos por 2 años y no sometidos a tratamiento, experimentan un cambio de peso promedio de 0,5 kilos $^{23}$.

Diversos estudios avalan que las intervenciones en estilo de vida logran mejorar los factores de riesgo cardiovascular en sujetos con obesidad ${ }^{3,4,20,24,25}$, resultado que en este estudio se observó en los grupos con menor exceso de peso inicial, sugiriendo que intervenciones tempranas tendrían un mayor impacto, siendo insuficiente el tratamiento médico en obesidad severa ${ }^{26,27,28}$. Otros estudios afirman que los pacientes con menor grado de obesidad inicial $\left(\mathrm{IMC}<35 \mathrm{~kg} / \mathrm{m}^{2}\right)$ requieren intervenciones menos intensivas y estructuradas que los sujetos con IMC $>35 \mathrm{~kg} / \mathrm{m}^{2}$, lo que indica que se debería considerarse el grado de obesidad para decidir el tipo de intervención y así administrar mejor los recursos ${ }^{29}$. Este estudio, al igual que otros, encontró que pequeñas disminuciones de peso $(\geq 5 \%$ del peso) logran reducir los niveles de glicemia, y por tanto, el riesgo de desarrollar diabetes ${ }^{6,8,20,30}$.

Dentro de las fortalezas de este estudio se encuentra el alto porcentaje de participantes que acudió a control al término del programa y el adecuado registro de los parámetros antropométricos. Al considerar las limitaciones de este trabajo, cabe mencionar en primer lugar que la elección de los centros analizados no fue al azar y que pertenecen a una comuna de clase socioeconómica media, lo cual podría no ser representativo de la realidad nacional y probablemente los resultados pudieran ser menos alentadores en comunas más vulnerables. En segundo lugar tenemos la falta de registro de los parámetros metabólicos y asistencia a talleres, lo que pudo provocar sesgo sobre los análisis y limitar la validez de las conclusiones. Problema similar ocurrió durante la evaluación nacional del PASAF realizada el 2008, donde se revisaron los datos de 285 establecimientos, pudiéndose analizar sólo 81 centros, ya que el resto carecía de información suficiente ${ }^{11}$. La escasa participación de hombres supone también una limitación.

En suma, en general los programas de intervención sobre obesidad en APS presentan baja adherencia y resultados modestos, lo cual hace necesario que se lleven a cabo evaluaciones nacionales de costo-efectividad que permitan decidir y justificar la pertinencia de estos programas como políticas públicas. Sería conveniente incluir evaluaciones a mediano y largo plazo, ya que es posible que resultados tan modestos sean difíciles de mantener a través del tiempo, pudiendo ser más efectivo dirigir estos esfuerzos a la población infantil.

\section{Conclusión}

El PASAF redujo levemente los parámetros nutricionales. La corrección de los parámetros 
metabólicos fue especialmente efectiva en los sujetos menos obesos y en los que redujeron un mayor porcentaje de peso. La asistencia registrada a los talleres y actividad física fue baja.

Agradecimientos: A los CESFAM pertenecientes a la comuna de Macul (Santa Julia, Félix de Amesti, Padre Hurtado) y a las nutricionistas a cargo del PASAF en dichos centros, Sras. Cora González, Elizabeth Cayunir y Alicia Bravo, respectivamente, por la valiosa información otorgada.

\section{Referencias}

1. Organización Mundial de la Salud. Mayo de 2012. Obesidad y sobrepeso. (Disponible en: http://www.who.int/ mediacentre/factsheets /fs311/es. Consultado en mayo de 2014).

2. Ministerio de Salud de Chile, Pontificia Universidad Católica, Universidad Alberto Hurtado. 2011. Encuesta Nacional de Salud ENS Chile 2009-2010.(Disponible en:http://web.minsal.cl/portal/url/item/bcb03d7bc28b64dfe040010165012d23.pdf. Consultado en marzo de 2014).

3. Wadden TA, Volger S, Sarwer DB, Vetter ML, Tsai AG, Berkowitz RI, et al. A two-year randomized trial of obesity treatment in primary care practice. N Engl J Med 2011; 365: 1969-79.

4. Jebb SA, Ahern AL, Olson AD, Aston LM, Holzapfel C, Stoll J, et al Primary care referral to a commercial provider for weight loss treatment versus standard care: a randomised controlled trial. Lancet 2011; 378: 1485-92.

5. Admiraal WM, Vlaar EM, Nierkens V, Holleman F, Middelkoop BJ, Stronks K, et al. Intensive lifestyle intervention in general practice to prevent type 2 diabetes among 18 to 60-year-old South Asians: 1-year effects on the weight status and metabolic profile of participants in a randomized controlled trial. PLoS One 2013; 8 (7): e68605.

6. Saaristo T, Moilanen L, Korpi-Hyövälti E, Vanhala M, Saltevo J, Niskanen L, et al. Lifestyle intervention for prevention of type 2 diabetes in primary health care: one-year follow-up of the Finnish National Diabetes Prevention Program (FIN-D2D). Diabetes Care 2010; 33: 2146-51.

7. Ministerio de Salud de Chile. Julio de 2008. Orientaciones Técnicas, Programa de Alimentación Saludable y Actividad Física para la Prevención de Enfermedades Crónicas en Niños, Niñas, Adolescentes y Adultos 20082009. (Disponible en: http://web.minsal.cl/portal/url/ ite m/69e15f0c31354025e04001011f0133d1.pdf. Consultado en marzo de 2014).

8. Carrasco F, Moreno M, Irribarra V, Rodríguez L, Martin MA, Alarcón A, et al. [Evaluation of a pilot intervention program for overweight and obese adults at risk of type 2 diabetes]. Rev Med Chile 2008; 136: 13-21.

9. Ministerio de Salud de Chile. Agosto de 2008. Minuta Ejecutiva, Programa de Tratamiento de la Obesidad: Alimentación Saludable y Actividad Física (en adultos, niños, niñas y adolescentes) y Obesidad Mórbida. Dirección de presupuesto.(Disponible en : http://www.dipres. gob.cl/574/articles-38659_doc_pdf.pdf. Consultado en marzo de 2014).

10. Ministerio de Salud de Chile. Julio 2010. Informe de Evaluación, Programa de Tratamiento de la Obesidad: Alimentación Saludable y Actividad Física (en adultos, niños, niñas y adolescentes) y Obesidad Mórbida. Departamento de Coordinación de Programas. Disponible en: http://www.marcologico.cl/pasaf/Admin/Archivos/ Informe $\% 20$ Pasaf $\% 202010 \% 20$ con $\% 20$ datos $\% 202009$. pdf. (Consultado en marzo de 2012).

11. Ministerio de Salud de Chile, Fondo Nacional de Salud. Agosto de 2008. Informe Final de Evaluación, Programa de Tratamiento de la Obesidad: Alimentación Saludable y Actividad Física (en adultos, niños y adolescentes) y Obesidad Mórbida. (Disponible en: http://www.dipres. gob.cl/574/articles-38653_doc_pdf.pdf. Consultado en marzo de 2014).

12. Ministerio de Salud de Chile. 31 de octubre de 2012. Informe de Cumplimientos de Compromisos al 30-062012. Programa de Tratamiento de la Obesidad. PASAF Adulto e Infantil y Obesidad Mórbida. (Disponible en: http://www.dipres.gob.cl/595/articles-94525_doc_pdf. pdf. Consultado en marzo de 2014).

13. Organización Mundial de la Salud. Marzo de 2013. Diez datos sobre la obesidad, clasificación del IMC. (Disponible en: http://www.who.int/features/factfiles/obesity/ facts/es/index.html.Consultado en mayo de 2014).

14. Evaluation of the Counterweight Programme for obesity management in primary care: a starting point for continuous improvement. Br J Gen Pract 2008; 58: 548-54.

15. Fuentes L, Muñoz A. Efectos de una intervención integral en la reducción de factores de riesgo cardiovasculares con sobrepeso u obesidad de la Región de Los Ríos. Rev Med Chile 2010; 138: 974-81.

16. Arrebola Vivas E, López Plaza B, Koester Weber T, Bermejo López L, Palma Milla S, Lisbona Catalán A, et al. Predictor variables for low adherence to a lifestyle modification program of overweight treatment in primary health care. Nutr Hosp 2013; 28: 1530-5. 
17. Klein S, Burke LE, Bray GA, Blair S, Allison DB, PiSunyer X, et al. Clinical implications of obesity with specific focus on cardiovascular disease: a statement for professionals from the American Heart Association Council on Nutrition, Physical Activity, and Metabolism: endorsed by the American College of Cardiology Foundation. Circulation 2004; 110: 2952-67.

18. Douketis JD, Macie C, Thabane L, Williamson DF. Systematic review of long-term weight loss studies in obese adults: clinical significance and applicability to clinical practice. Int J Obes (Lond) 2005; 29: 1153-67.

19. U.S. Food and Drug Administration. February 2007. Guidance for industry. Developing products for weight management. (Disponible en: http://www.fda.gov/ downloads/Drugs/GuidanceComplianceRegulatoryInformation/Guidances/ucm071612.pdf. Consultado en marzo de 2012).

20. Tuomilehto J, Lindstrom J, Eriksson JG, Valle TT, Hämäläinen $\mathrm{H}$, Ilanne-Parikka $\mathrm{P}$, et al. Prevention of type 2 diabetes mellitus by changes in lifestyle among subjects with impaired glucose tolerance. $\mathrm{N}$ Engl J Med 2001; 344: 1343-50.

21. Pimenta GP, Saruwatari RT, Correa MR, Genaro PL, Aguilar-Nascimento JE. Mortality, weight loss and quality of life of patients with morbid obesity: evaluation of the surgical and medical treatment after 2 years. Arq Gastroenterol 2010; 47: 263-9.

22. Csendes A, Burdiles P, Papapietro K, Burgos AM. [Review of the results of medical and surgical treatment of morbid obesity]. Rev Med Chile 2009; 137: 559-66.

23. Bennett GG, Warner ET, Glasgow RE, Askew S, Goldman J, Ritzwoller DP, et al. Obesity treatment for so- cioeconomically disadvantaged patients in primary care practice. Arch Intern Med 2012; 172: 565-74.

24. Artinian NT, Fletcher GF, Mozaffarian D, Kris-Etherton P, Van Horn L, Lichtenstein AH, et al. Interventions to promote physical activity and dietary lifestyle changes for cardiovascular risk factor reduction in adults: a scientific statement from the American Heart Association. Circulation 2010; 122: 406-41.

25. Appel LJ, Clark JM, Yeh HC, Wang NY, Coughlin JW, Daumit G, et al. Comparative effectiveness of weightloss interventions in clinical practice. $\mathrm{N}$ Engl J Med 2011; 365: 1959-68.

26. Bambs C, Cerda J, Escalona A. Morbid obesity in a developing country: the Chilean experience. Bull World Health Organ 2008; 86: 813-4.

27. Carrasco F, Klaassen J, Papapietro K, Reyes E, Rodríguez L, Csendes A, et al. [A proposal of guidelines for surgical management of obesity]. Rev Med Chile 2005; 133: 699706.

28. Carrasco F, Manrique M, De la Maza MP, Moreno M, Albala C, García J, et al. [Statement about the medical and surgical treatment of overweight and obesity]. Rev Med Chile 2009; 137: 972-81.

29. Azar KM, Xiao L, Ma J. Baseline obesity status modifies effectiveness of adapted diabetes prevention program lifestyle interventions for weight management in primary care. Biomed Res Int 2013; 2013: 191-209.

30. Leblanc ES, O'Connor E, Whitlock EP, Patnode CD, Kapka T. Effectiveness of primary care-relevant treatments for obesity in adults: a systematic evidence review for the u.s. Preventive services task force. Ann Intern Med 2011; 155: 434-47. 